\title{
LE RÔLE DES INSTITUTIONS CULTURELLES ET DES MISSIONS À L'ÉTRANGER DANS LA CIRCULATION DES IDÉES GÉOGRAPHIQUES. L'EXEMPLE DE LA CARRIÈRE DE PIERRE DEFFONTAINES (1894-1978)
}

Claire Delfosse ${ }^{1}$

Résumé: Durant l'entre-deux-guerres la France renforce sa politique culturelle à l'étranger. Elle multiplie la création d'Instituts français, en particulier dans les pays de la Petite Entente et l'Amérique latine. Ces instituts, véritables têtes de pont à proximité des principales universités étrangères permettent d'envoyer des universitaires en mission. Pierre Deffontaines (1894-1978) est une personnalité très particulière dont l'essentiel de la carrière s'est déroulée à l'étranger. A partir de ses deux principaux postes - l'un en France aux Facultés catholiques de Lille (19241939) et l'autre à l'étranger (en tant que directeur de l'Institut français de Barcelone de 1939 à 1964) - il effectue de nombreuses missions à l'étranger, en particulier dans les deux Amériques: Brésil, Argentine, Uruguay et Québec. Il serait le géographe français de sa génération le plus connu à l'étranger et celui qui aurait créé le plus de départements de géographie et d'associations géographiques à l'étranger. Disciple de Jean Brunhes, il réussit à associer la promotion de la culture française à la promotion de la géographie.

Mots-clés: Instituts français, mission à l'étranger, Pierre Deffontaines, Facultés catholiques de Lille, Brésil, Barcelone.

\begin{abstract}
THE PlaCE OF CUlTURAL POLICY ABROAD IN THE CIRCULATION OF GEOGRAPHICAL IDEAS: THE CASE OF THE CAREER OF PIERRE DESFONTAINES (1894-1978) - Between the two world wars, France reinforced its cultural policy abroad. Many French Institutes were founded, particularly in the countries of the Petite Entente and in Latin America. These institutes were located near foreign universities which helped French Scholars travel abroad. Pierre Deffontaines (1894-1978) is significant in this respect, as most of his career took place abroad. Thanks to the two main successive positions which he held - one in France at the Catholic Faculties of Lille (1924-1939), the other in Spain as the director of the French Institute of Barcelona (1939-1964) he travelled extensively abroad, particularly in the Americas: Brazil, Argentina, Uruguay and
\end{abstract}

1 Maître de Conférences à l'Université de Lille I (Laboratoire de Géographie humaine) et membre du laboratoire CNRS-EHGO (Paris). UFR Geographie, Boulevard P. Langevin. Université des Sciences et Technologies de Lille. 59655 Villeneuve-d'Ascq cedex, France.

Tel.: (33)(0)3 203360 83; Fax: (33) (0)3 203360 74; E-mail: Claire.Delfosse@ univ-lille1.fr 
Québec. He may have been the French geographer who was the best known abroad and who promoted the largest number of geography departments and associations in various places. As a disciple of Jean Brunhes, he succeeded in associating the promotion of French culture to the promotion of geography.

Key-words: French institutes, cultural policy abroad, Pierre Deffontaines, Catholic Faculties of Lille, Brasil, Barcelona.

Resumo: O PAPEL DAS INSTITUIÇÕES CULTURAIS E DAS MISSÕES NO ESTRANGEIRO NA CIRCULAÇÃO DAS IDEIAS GEOGRÁFICAS. O EXEMPLO DA CARREIRA DE PIERRE DEFFONTAINES (1894-1978) - No período entre as duas guerras a França reforçou a sua política cultural no estrangeiro, criando vários institutos franceses, em particular nos países da "Petite Entente" e na América Latina. Estes institutos, criados perto de universidades estrangeiras, facilitaram as comissões de serviço no estrangeiro de estudiosos franceses. Pierre Deffontaines (1894-1978) é uma figura particular da geografia francesa, cuja carreira se desenvolveu em grande parte no estrangeiro. Graças a dois dos seus cargos - em França, nas Faculdades Católicas de Lille (1924-1939), e em Barcelona como director do Instituto Francês, entre 1939 e 1964 - fez várias viagens ao estrangeiro, sobretudo à América: Brasil, Argentina, Uruguai, Quebec. Foi o geógrafo francês, da sua geração, mais conhecido no estrangeiro e aquele que criou mais departamentos, sociedades geográficas e associações em vários países. Discípulo de Jean Brunhes conseguiu aliar a promoção da cultura francesa à da geografia.

Palavras-chave: Institutos franceses, política cultural no estrangeiro, Faculdades Católicas de Lille, Pierre Deffontaines, Brasil, Barcelona.

Durant l'entre-deux-guerres la France renforce sa politique culturelle à l'étranger. Elle multiplie la création d'Instituts français, en particulier dans les pays de la Petite Entente et l'Amérique latine. Ces instituts, véritables têtes de pont à proximité des principales universités étrangères (CHARLE, 1994) permettent dans un deuxième temps d'envoyer des universitaires en mission, voire parfois en postes fixes pour quelques années. Les géographes vont être partie prenante de cette politique et l'on trouve parmi les premiers à y souscrire Emmanuel de Martonne. Une fois en mission ces géographes sont-ils seulement des ambassadeurs de la culture française ou contribuent-ils par ailleurs à la diffusion des savoirs géographiques?

Pierre Deffontaines (1894-1978) est une personnalité très particulière dont l'essentiel de la carrière s'est déroulée à l'étranger. A partir de ses deux principaux postes - l'un en France aux Facultés catholiques de Lille (1924-1939) et l'autre à l'étranger (en tant que directeur de l'Institut français de Barcelone de 1939 à 1964) - il effectue de nombreuses missions à l'étranger, en particulier dans les deux Amériques: Brésil, Argentine, Uruguay et Québec. Il serait le géographe français de sa génération le plus connu à l'étranger et celui qui aurait créé le plus de départements de géographie et d'associations géographiques à l'étranger. 


\section{1 - DEFFONTAINES, UN HOMME DE RÉSEAUX}

Pierre Deffontaines à l'instar de la plupart des géographes français des années 1920-1950, qui ont fait carrière à l'étranger, a deux caractéristiques, celle d'être marginal au sein de l'Université française et celle d'être un homme de réseaux.

\section{1 - "Le réseau catholique"}

Au début du $\mathrm{XX}^{\mathrm{e}}$ siècle, les affiliations idéologiques jouent un grand rôle dans le choix et la poursuite d'une carrière (BERDOULAY, 1995). En effet, la plupart des universitaires qui ont alors des chaires en France, sont des intellectuels ralliés à la République et sont le plus souvent laïques; la République reste méfiante envers les catholiques pratiquants. Or, pour ceux-ci, dans certains pays où l'influence française s'est appuyée dans un premier temps sur des congrégations religieuses, ou qui sont fortement marqués par la puissance de l'église catholique comme le Québec, la Pologne, l'Espagne, la religion devient alors un atout pour asseoir la présence française. Pierre Deffontaines illustre parfaitement cette caractéristique. En effet, Deffontaines est un fervent catholique, un catholique rallié à la République et de la tendance que l'on appelle le catholicisme social. Pendant son séjour à la fondation Thiers (sorte de bourse de thèse pour les agrégés), de 1922 à 1925, il rencontre Robert Garric, initiateur d'un mouvement de culture pour tous, les Equipes sociales, et s'implique fortement dans ce mouvement. Il y côtoie d'autres universitaires et chercheurs qui seront eux aussi marginalisés dans leur carrière française et qui constitueront un réseau de catholiques faisant carrière à l'étranger.

On peut supposer que cette forte implication catholique ne lui a pas permis d'obtenir une chaire à l'Université après l'agrégation. Faisant partie de cette génération d'universitaires qui, après 1920, éprouve quelques difficultés à obtenir des chaires à l'Université, il accepte de fonder et de tenir la chaire de géographie aux Facultés catholiques de Lille (1924-1938).

Dans cette région où le mouvement catholique social est important il demeure très actif. Il participe, avec le Père Chatelain et Louise Taphanel, à la fondation de "l'Union des Trois Ordres" (UTO). Cette Union cherche à lutter contre la trop grande imperméabilité entre le primaire, le secondaire, et l'enseignement supérieur. Il a donc une conception unitaire de l'enseignement en particulier de celui de la géographie. Dans sa pédagogie, les voyages, la découverte des paysages et de la nature jouent un grand rôle. "Je me suis longuement occupé d'une pédagogie de l'observation géographique", note-t-il dans son autobiographie. C'est ainsi qu'il publie pour les scouts de France un Guide du voyageur actif qui connaîtra un véritable succès (1938). Il noue aussi des liens avec le milieu de l'édition, en particulier catholique, puis avec Gaston Gallimard chez qui il dirigera la collection de géographie humaine.

Son milieu familial et les réseaux de relations issus de sa famille jouent un rôle important dans sa carrière. Il est le fils du premier général tué durant la guerre de 1914. Comme bon nombre d'intellectuels de sa génération, il ressent une grande admiration 
pour Pétain, avec tout ce que cela comporte de déchirements au moment de la période de Vichy. Il a toujours l'idée, que quoiqu'il arrive, il faut servir la France. A la suite de l'invasion du Nord de la France par les Allemands, sa famille part pour le Limousin où il fait ses premières recherches préhistoriques. C'est là qu'il lit ses premiers ouvrages de géographie et notamment la géographie humaine de Jean Brunhes et c'est par ses relations familiales qu'il rencontre celui qui sera son maitre, Jean Brunhes.

Deux autres membres de sa famille, ses beaux-frères, jouent un rôle important dans l'évolution de sa carrière: Roger Pons, professeur de littérature en khâgne puis inspecteur de l'enseignement supérieur, pour ses théories pédagogiques et Auguste Viatte, professeur de littérature française et qui a, lui aussi, fait l'essentiel de sa carrière à l'étranger.

\section{2 - Un parcours universitaire original}

Du point de vue de son "parcours géographique" il cumule tous les handicaps. Il n'est pas passé par le système scolaire, il a travaillé avec des précepteurs et comme une sorte d'autodidacte. Il n'arrive pas à la géographie directement (ou par l'histoire), mais a fait une licence de Droit (pour répondre aux vœux de son père) puis s'est intéressé à la préhistoire. Il a découvert la géographie à travers la lecture de Jean Brunhes (18691930) qui restera son maître et père adoptif comme il l'écrit par la suite. Il n'est pas normalien.

A partir de sa rencontre avec Jean Brunhes en 1918, il décide de s'orienter principalement vers la géographie. Il suit alors ses cours au Collège de France et ceux de Lucien Gallois, d'Albert Demangeon, d'Emmanuel de Martonne à la Sorbonne. Il passe une licence d'histoire et géographie et un DES (actuelle maîtrise), sous la direction de Demangeon. Il passe son agrégation d'histoire-géographie en 1922 et obtient pour trois ans la bourse Thiers sur l'intervention de Jean Brunhes (1922-1925). Mais il n'abandonne pas pour autant sa passion pour la préhistoire: il étudie aussi à l'Institut de Paléontologie humaine et obtient un diplôme de l'école du Louvre mention spéciale préhistoire en 1920. Son DES de géographie porte sur la géographie préhistorique (Essai de géographie préhistorique du Limousin et de son pourtour sédimentaire). Il se constitue un réseau important parmi les préhistoriens et anthropologues et est ainsi très ami de Leroi-Gourhan, avec qui il fonde, quelques années plus tard, en 1958, l'éphémère Revue de géographie et d'ethnographie.

A partir de 1924, une fois à l'Université catholique de Lille, il devient aussi un homme des sociétés de géographie. Il participe régulièrement à la vie de la Société de géographie de Lille dont Max Sorre assure le secrétariat général et collabore régulièrement depuis 1922 à son Bulletin. Après le départ de Sorre en 1931, il en reprend le secrétariat général jusqu'en 1937. Dans le même temps, de 1935 à 1938, il est membre de la Commission exécutive de la Société de géographie de Paris. De cette période naît aussi son goût pour les congrès de l'UGI. Il est à celui de Paris en 1931, puis à Amsterdam en1938. Il participe aussi aux congrès internationaux d'anthropologie.

Sa pensée sera fortement marquée par cette formation un peu "éclectique", mais 
surtout par son souci d'appréhension de l'homme et du devenir de l'humanité, devenir qu'il voit selon les théories de Teilhard de Chardin. Sa pensée géographique est surtout marquée par celle de son maître Jean Brunhes dont il a poursuivi un certain nombre d'œuvres. Le fait d'être l'élève et le disciple de Jean Brunhes, enseignant au Collège de France et non à la Sorbonne, institutions plus rivales que complémentaires alors, ne fait qu'accroître son caractère marginal. Une thèse peu conforme et dédiée à Saint François d'Assise ne lui a sûrement pas permis l'obtention d'une chaire dans une Université française après Lille. Il n'aura donc pas de poste dans une Université française mais à l'Institut français de Barcelone.

De Lille, il fait également des voyages dans les pays de la Petite Entente, sur les traces de de Martonne, qu'il cite abondamment. Puis, à partir de 1935, il va aller régulièrement en mission en Amérique latine et au Québec.

\section{2 - DEFFONTAINES, ADMINISTRATEUR, AMBASSADEUR DE LA CULTURE FRANÇAISE OU DE LA GÉOGRAPHIE?}

L'essentiel de sa carrière, Pierre Deffontaines le fait à l'étranger. Certes, ce n'est pas un grand théoricien, il a la plume facile et un style qui se rapproche plus de celui des explorateurs que de celui des scientifiques, mais est-ce un défaut pour enseigner à l'étranger? Il semble appartenir à ce que Charle nomme la catégorie des "ambassadeurs": "les professeurs qui s'adressent de préférence à un public large auquel ils présentent la culture générale au sens français plutôt que la science en train de se faire". En tant que pédagogue chevronné et qui milite contre la séparation entre le second cycle et l'Université, il ne s'offusque pas, contrairement à d'autres universitaires, de devoir enseigner à un public qui ressemble à celui des écoles normales en France. Enfin, de part son action sociale, il a aussi des qualités d'organisateur; qualités précieuses pour asseoir l'enseignement universitaire d'une nouvelle discipline ainsi que sa diffusion. Sa pratique des milieux de l'édition lui est également utile à l'étranger pour contribuer à la publication de travaux géographiques.

\section{1 - Deffontaines au Brésil, l'administrateur au service de la géographie}

Dans l'entre-deux-guerres, un fort mouvement de rénovation universitaire part de l'Argentine pour gagner les autres pays de l'Amérique latine. Ce mouvement vise, entre autres, à réconcilier l'Université avec la vie concrète, ainsi qu'avec la culture et la civilisation de l'Amérique latine. L'histoire, la géographie, l'anthropologie ont un grand rôle à jouer dans ce mouvement de rénovation. La France, qui cherche à renforcer ses relations avec l'Amérique latine et qui a souvent accueilli les élites des Etats d'Amérique latine, est sollicitée pour aider à établir ces Universités nouvelles.

A la Sorbonne, deux personnages-clé s'occupent des relations avec les Universités d'Amérique latine: Martinenche, un hispaniste et Georges Dumas, philosophe et psychologue. En 1934, l'Université de São Paulo est fondée et fait appel à des universitaires français. Deffontaines fait partie de l'équipe française chargée de créer un certain 
nombre d'enseignements à la nouvelle Université. Il a été signalé à Georges Dumas, par Robert Garric. Il accepte et part en 1934 pour les débuts de l'année scolaire brésilienne. Il va jouer un rôle prépondérant pour la création de la chaire de géographie, qui est "chargée de former pour le Brésil et plus particulièrement pour l'Etat de Saint-Paul une équipe de jeunes géographes qui s'attacheront aux problèmes du pays, problèmes physiques et humains." (DEFFONTAINES, Bulletin de la Société de géographie de Lille, 1935). Pour établir cette chaire, il fonde à São Paulo une Association des géographes brésiliens, dont les deux premiers présidents furent français (Deffontaines, puis Monbeig). Pour ce faire, il réunit chez lui quelques personnes dont aucune n'était géographe, mais des notables locaux tous francophiles (MONBEIG, in Hommage à Pierre Deffontaines, 1979). Il s'appuie donc sur le modèle des sociétés de géographie françaises qui avaient permis l'institutionnalisation de la géographie en France.

La position de la géographie universitaire et l'Association des géographes brésiliens vont se renforcer par l'action qu'il va entreprendre lors de nouvelles missions à Rio de Janeiro (en 1936-37 et 1938). Là, il va contribuer avec de Martonne à la fondation de l'Institut de géographie et de statistique de l'Université de Rio de Janeiro et à l'organisation du conseil national de géographie du Brésil, section brésilienne du comité international de géographie. Il contribue aussi à fonder des revues de géographie brésilienne: le Bulletin de l'Association des Géographes et la Revista brasileira de geografia.

En acceptant le poste de Barcelone il relève un défi, tout en continuant à partir en missions en Amérique latine (Argentine, Uruguay, Brésil en 1946; Pérou, Bolivie, Equateur, Colombie en 1950; Mexique, Haiti, 1954; Chili, 1959), ainsi qu'au Québec, 1948) où il interviendra dans le même esprit qu'au Brésil (HAMELIN, in Hommage à Pierre Deffontaines, 1978 et 1986).

2.2 - Deffontaines en Catalogne, ambassadeur de la culture française ou promoteur de la géographie catalane?

Juste avant la Seconde Guerre mondiale, en 1939, et après la guerre civile espagnole, on lui demande d'aller réinstaller l'Institut français de Barcelone. "C'était une mission difficile, au milieu d'un pays ruiné et affamé et avec le handicap que la France n'avait pas joué la carte franquiste et était très mal vue", note-t-il dans son autobiographie. Mais une mission difficile dont il s'est certainement bien acquitté car il restera un quart de siècle à la tête de l'Institut français de Barcelone.

Deffontaines a plusieurs atouts pour être nommé à Barcelone, son expériences d'organisateur à Lille puis à São Paulo et à Rio et des qualités de "diplomate". Il est issu d'une famille amie de Pétain qui était alors ambassadeur à Madrid; en tant que catholique il répond aux moyens fixés par le gouvernement français pour rétablir la présence culturelle et scolaire en Espagne: tenter une action originale de rapprochement spirituel par la religion catholique. En Espagne il se retrouve avec le géographehistorien-ethnographe Maurice Legendre responsable de la politique culturelle, et avec Paul Guinard chargé de remettre en route les Instituts français en Espagne à partir de 
Madrid, dont les profils sont similaires au siens: Maurice Legendre avait soutenu une thèse fortement marquée par la pensée de Brunhes.

Sa tâche à Barcelone sera difficile: en 1942, tout comme les directeurs des Instituts de Madrid et de Lisbonne ainsi que leurs enseignants, il ne fait pas acte d'allégeance au gouvernement de Vichy. Avec toute son équipe de professeurs de l'Institut français de Barcelone il est chassé par la police espagnole, sur l'ordre du consul général de Vichy. Il organise alors, avec l'aide de Catalans, un nouvel Institut français attaché au gouvernement d'Alger. Cet Institut a vécu clandestinement durant un an et demi sous le nom de l'ANEIE (Association nationale d'étudiants d'idiomes étrangers), n'ayant plus le droit de s'appeler ni Institut ni français. De véritables liens se nouent avec les intellectuels catalans: si ceux-ci aident Deffontaines à braver le gouvernement de Vichy que soutient Franco, lui-même aide les Catalans à exprimer leur culture opprimée par le régime franquiste. L'Institut français de Barcelone devient une sorte d'îlot de liberté de parole. Les livres français qu' on y trouve contribuent aussi à "détourner" la censure franquiste.

Mais Pierre Deffontaines, directeur de l'Institut français de Barcelone, n'est-il qu' ambassadeur de la culture française, de la France libre, ou va-t-il aussi œuvrer pour la géographie? Ici aussi, il fait œuvre de géographe. Il arrive en Catalogne alors que la géographie espagnole est décapitée par l'arrivée du franquisme: le géographe catalan, chef de file de l'école espagnole, Pau Vila est en exil en Argentine. Vilà Valentí note que, vers 1945-1950 et immédiatement après, seule une poignée de géographes à la Société catalane de géographie pouvait préserver et entretenir le souffle de la géographie catalane qui n'avait pas encore pu prendre toute sa force pendant les années qui précédèrent la guerre civile. Pierre Deffontaines aida ce petit groupe auquel se rattachèrent quelques étudiants à l'Université. Là aussi, il joue son rôle d'homme d'association, en organisant le Cercle des géographes de l'Institut français: des professeurs catalans, des spécialistes de disciplines voisines, quelques professeurs français et ceux qui entreprenaient des enquêtes géographiques, exposaient là les résultats de leurs travaux. Les livres de géographie disponibles à la bibliothèque de l'Institut, les conférences faites par lui-même et par d'autres géographes français qu'il faisait venir, jouent un rôle fondamental dans la renaissance de la géographie catalane. D'ailleurs il donne aussi des cours à l'Université de Barcelone et au Conseil Supérieur des Recherches Scientifiques (Institut Elcano).

Ainsi, dans son simple rôle d'ambassadeur de la culture française, il va faire œuvre de géographe, à tel point que, si en France on ne lui connaît pas de disciples, ce n'est pas le cas en Catalogne. Josefina Gomez Mendoza (1997) montre qu'il a eu une influence décisive en matière de méthode; une influence que Llobet dans sa thèse sur le Montseny reconnaît dès l'introduction (1946). Il contribue surtout à développer la géographie humaine, dans une Espagne jusqu'alors beaucoup plus tournée vers la géographie physique. 
Ainsi, Pierre Deffontaines note lui-même à plusieurs reprises dans son autobiographie combien, pour lui, promotion de la culture française et promotion de la géographie sont associées:

A propos de ses missions au Brésil: "Cela m'orienta définitivement vers les pays d'Amérique latine où je fus chargé d'une série de missions les années suivantes [...]. J'y renforçais à la fois l'influence française et celle de la géographie, exerçant un véritable apostolat géographique par les nombreux articles que j'y publiais et par les conférences que j'y faisais."

Et à propos de la Catalogne: "Je continuai mon système qui consistait à utiliser la géographie comme élément de propagande à la fois française et géographique."

\section{3 - DEFFONTAINES: UN GÉOGRAPHE PIONNIER}

Pierre Marthelot, un ancien compagnon des équipes sociales et Directeur d'études à l'EHESS, dans la note nécrologique qu'il consacre à Pierre Deffontaines (Acta geographica, 1979), le qualifie de pionnier de la recherche et de l'enseignement de la géographie. "A chaque fois, il fonde, il enseigne, il observe; mais en même temps, il fait équipe, se refusant à l'indifférence hautaine, refuge de trop d'entre nous, et le fluide passe, courant d'humanité, consubstantiel au savoir et à sa diffusion ". Pionnier il l'est en effet à plusieurs titres.

\section{1 - Le fondateur géographe}

On peut le considérer comme un pionnier au niveau de la transmission des savoirs géographiques: par la fondation de chaires et des outils nécessaires à leur pérennisation (comme les associations). Au-delà du rôle administratif et de la présence universitaire française, il a contribué à enraciner la géographie française dans les deux Amériques (PINCHEMEL, in Hommage à Pierre Deffontaines, 1979). Ses postes et missions ont certes un aspect administratif important, mais il enseigne aussi. Il diffuse la méthode de l'excursion et, de ce fait, apprend peut-être à voir avec l'œil et les méthodes du géographe français. Un témoin des excursions qu'il a organisées lorsqu'il était à l'Université Laval raconte "nous apprîmes que la géographie était la science du paysage, de la terre humanisée" (LAVAL, in Hommage à Pierre Deffontaines, 1978). Pierre Monbeig souligne également l'importance de son action au Brésil. "Il n'est sans doute pas excessif de dire qu'avant son arrivée à l'Université de São Paulo en 1934, la géographie brésilienne était quasiment inexistante" (Acta geographica, 1979). Mais, quelques mois plus tard, l'élan était donné:

"ses conférences avaient attiré l'élite intellectuelle de la capitale du café; elle eut ainsi la révélation de l'art de voyager, de ce que contient un paysage et de ce qu'est la géographie française la plus authentique. Il entraîna sur les routes et les pistes de l'État de São Paulo un petit noyau d'étudiants et d'amis; il leur apprit les charmes de la marche à pied pour mieux découvrir leur propre pays. " (Acta geographica, 1979).

L'importance qu'il accordait à la méthodologie est également soulignée par Vilà 
Valentí qui était assidu à ses conférences à Barcelone (1979)

Il a donc joué un rôle non négligeable dans la diffusion des méthodes de la géographie française dans les pays étrangers, mais il est aussi un ambassadeur de la géographie et du voyage pour un très large public: son Guide du voyageur actif édité pour les scouts en est un exemple, ainsi que quelques articles sur la façon d'aborder la nature ("Vers un nouveau tourisme... à l'exemple d'Arthur Young", Bull. de la Société de Géographie de Lille, 1929 ou "Comment connaître et comprendre un coin de pays, ou petit guide du voyageur actif pour les vacances", ou encore "Conseils pour la découverte d'un coin de nature", 1937). Il a su diffuser les savoirs géographiques auprès du grand public en collaborant auprès de revues comme La Revue des Deux Mondes ("Les personnages au Brésil", 1936) ou en écrivant des ouvrages de vulgarisation à caractère touristique comme celui qu'il a consacré à l'Espagne, en collaboration avec Duriat, un historien de l'art: Catalogne, Baléares, Levant (1957, Paris, Arthaud). Il a également collaboré à l'Encyclopédie de l'Amérique latine (1954, Presses Universitaires de France), destinée à mieux faire connaître ce continent aux Français. Enfin, il peut être considéré comme un ardent promoteur de la géographie et d'une géographie humaine mondiale et humaniste, comme le note Philippe Pinchemel, avec sa collection La géographie humaine chez Gallimard et les volumes de la Pléiade consacrés à la géographie qu'il a dirigé (1966 et 1975).

Toujours homme de réseaux, d'association et d'édition, il a contribué à fonder des revues à l'étranger, comme les revues brésiliennes, ou à soutenir les nouvelles revues comme celle de l'Institut de géographie de Barcelone ou en France les revues des Universités régionales qui portent sur l'Espagne (Méditerranée, par exemple) ou sur l'Amérique latine (Cahiers d'Outre-Mer, Université de Bordeaux).

3.2 - Pionnier par la découverte de nouveaux terrains: au-delà de la monographie régionale française

Pierre Deffontaines est, comme ceux qui l'ont précédé et suivi, pionnier par la découverte de nouveaux terrains pour une géographie française qui, dans l'entre-deuxguerres, restait marquée par la monographie régionale en France (faible nombre de thèses à l'étranger). Son premier article sur le Brésil, publié dans le Bulletin de la Société de Géographie de Lille, est aussi particulièrement intéressant car il montre l'émerveillement du géographe face à la découverte d'un nouveau terrain.

"Le Brésil paraît devoir être une terre d'élection pour la Géographie. L'immensité du territoire, les connaissances encore imparfaites sur la majorité du pays et surtout l'extraordinaire rapidité des changements survenus, changements démographiques, sociaux, économiques et aussi physiques, par les déboisements et reboisements, par les endiguements ou les irrigations, orientent vers cette science." (1935)

D'ailleurs il note dans son autobiographie, à propos de sa première mission au Brésil:

"Ce fut tout de suite la grande découverte d'une géographie vierge que je faisais avec la collaboration active et enthousiaste d'une équipe d'étudiants aussi passionnés 
que moi d'apprendre la géographie nouvelle de leur propre pays."

Ses missions et son poste à Barcelone marquent fortement ses publications. Ainsi, dès 1935, il publie une première série d'articles sur le Brésil, puis alternent des publications sur le Québec, l'Espagne (en particulier la Catalogne et les îles Baléares) et l'Amérique latine. Il publie ainsi des ouvrages ou articles qui font référence dans le pays où il se trouve (Geografia humana do Brasil, 1952, Rio de Janeiro ou El Mediterraneo. Estudio de geografia humana, 1948, Barcelone) ou en France comme L'homme et l'hiver, ses travaux sur le "rang" ou l'ensemble de ses écrits sur l'élevage dans les pays d'Amérique du sud (Contribution à la géographie pastorale de l'Amérique latine. 1964, Rio de Janeiro).

\section{3 - Pionnier par la méthode qu'il adapte au nouveau terrain}

Les géographes en mission ou en poste à l'étranger reproduisent-ils à l'identique les théories de l'école de géographie française (V. BERDOULAY et SENECAL, "Blanchard au Québec: continuité ou rupture, in CLAVAL, 1995)? Les modèles classiques de monographies régionales mettaient l'accent sur les habitudes, les mœurs traditionnelles d'une communauté identifiée à un territoire en tenant compte d'une échelle de temps historique. La méthodologie de base de ces études consistait à bâtir une chaîne explicative à travers des plans structuraux descriptifs. Dans les pays "neufs" comme le Brésil et le Canada, il est difficile de dessiner les contours des genres de vie comme l'écrit Monbeig: "plus qu'une étude des genres de vie, c'est celle d'une société en mouvement que j'ai cherché à faire" (in H. THERY et al., 1991). D'ailleurs Raoul Blanchard note dans La géographie française au milieu du XXe siècle (1957), que Deffontaines a conduit une étude très nouvelle sur le "rang", mode de colonisation du territoire québécois ("Le peuplement du Canada français par le rang". Cahiers de l'Information géographique, 1952). Pierre Deffontaines va aussi être amené à dépasser la classification des habitations rurales de Demangeon dans l'Homme et la maison (1972), avec une conception proche de l'analyse systémique.

Un fait important caractérise la géographie des "géographes en mission", elle est ouverte aux autres disciplines: les échanges interdisciplinaires à l'étranger sont particulièrement forts et sont soulignés par Pierre Monbeig et Pierre Deffontaines et cela alors qu'en France, à partir de 1940, on note une sorte de repli disciplinaire. C'est peut-être aussi en cela que les géographes "à l'étranger ont été des pionniers": ils ont su s'approprier les nouveaux apports des autres disciplines.

On peut aussi se demander s'il n'y a pas d'échanges entre enseignants en mission et géographes locaux et en quoi ces derniers peuvent aussi contribuer à faire évoluer les méthodes et concepts des premiers. Cette question mériterait des études approfondies. Ce dont on est sûr c'est que les géographes français en mission ont contribué à faire éditer en France des travaux de géographes des pays où ils se trouvent ou avec lesquels ils sont en contact. Ainsi Pierre Deffontaines fait publier et préface l'ouvrage du canadien Benoît Brouillette, La chasse des animaux à fourrure au Canada, (1934). Pierre Deffontaines fait aussi des conférences sur la géographie de l'Amérique du Sud 
ou du Canada à Barcelone et initie des recherches sur l'Amérique latine alors qu'il est au Québec (1978). C'est encore un moyen de diffuser les connaissances géographiques et peut-être les savoirs. De même, si les rivalités entre pays pour l'influence intellectuelle dans le cadre de missions est grande (entre la France et les Etats-Unis par exemple en Amérique latine), il reste que les universitaires en poste ou en mission de différents pays s'y rencontrent et qu'on peut supposer qu'ils prennent connaissance de leur travaux et méthodes respectifs.

\section{4 - Un personnage ambigu}

Ainsi les missions et postes à l'étranger constituent, comme les congrès de l'UGI (ROBIC et al., 1996), un mode de construction de la communauté internationale géographique; les géographes "pionniers" y jouent un rôle prépondérant. Toutefois leur mission ou "ambassade" n'est pas toujours facile à analyser et se prête à des interprétations différentes suivant que l'on considère leur rôle dans leur pays d'origine ou de mission. Il est clair que le rôle de ces personnes mandatées par un gouvernement pour mettre en place les chaires de géographie à l'étranger, peut donner aux géographes locaux l'impression d'être dépossédés. En effet, ces derniers ne sont, dans un premier temps, que les assistants des universitaires français.

D'autre part, il y a ambiguïté sur les conditions dans lesquelles ils interviennent. En effet, les appuis universitaires français sont souvent sollicités par des Etats en quête d'identité nationale: ils cherchent à travers l'histoire et la géographie et l'institutionnalisation d'études universitaires non seulement à affirmer leur identité et les limites de leur territoire pour leur propre peuple mais aussi pour s'affirmer face à la communauté internationale. La géographie a donc là un rôle politique. Or, les Etats qui ont cette demande dans le courant des années 1930 à 1950 sont souvent des états fascisants. Dans ces conditions, des personnages comme Deffontaines, homme de réseau, catholique pratiquant...peuvent être considérés à posteriori comme des cautions ou comme étant à la solde d'Etats fascistes et persécuteurs. Il est vrai que Deffontaines a été appelé en Catalogne au moment où Pétain était ambassadeur de France, on dit aussi qu'il aurait été appelé au Québec pour faire contrepoids à l'influence de Raoul Blanchard qui n'était pas catholique, que, dans le Japon des années 1950, on a préféré diffuser les écrits de Deffontaines plutôt que ceux de Pierre George qui était communiste...

Cette ambiguïté est sûrement inhérente à la politique culturelle française ainsi qu'au rôle identitaire que l'on fait jouer à la géographie car rien dans la vie ni dans l'œuvre de Pierre Deffontaines ne laisse supposer qu'il ait versé dans le fascisme. C'était un idéaliste qui croyait en l'homme et au progrès humain. "La géographie humaine marque un accroissement de la mainmise des hommes sur la terre, un élargissement de la puissance de ceux-ci." (1948)

\section{BIBLIOGRAPHIE}




\section{Sources}

Historico da criação do Conselho Nacional de Geografia (1939). Revista Brasileira de Geografia: 9-18.

Autobiographie de Pierre Deffontaines, document dactylographié non daté, de 10 p.

La Catalogne vue par un géographe (1960) (célébration de la $20^{\text {ème }}$ année de présence de Deffontaines en Catalogne). Talleres gráficos Salve y T.G. Soler, Barcelone.

Hommage à Pierre Deffontaines (1894-1978) (1978). Cahiers de Géographie du Québec: 437-444.

Hommage à Pierre Deffontaines (1979). Acta Geographica, Société de Géographie, Paris: 3-24.

Migliorini, P. (1978) - Pierre Deffontaines. Bollettino Della Societa Geographica Italiana: 401-406.

Ouvrages de références

Collectif (1993) - Jean Bruhnes. Autour du monde, regards d'un géographe, regards de la géographie, Musée Albert Kahn.

BERDOUlay, V. (1995) - La formation de l'école française de géographie (1870-1914), Ed. du CTHS ( 2 édition), Paris.

BRoc, N. (1997) - Les géographes français en Espagne (1920-1950). Annales de Géographie, Paris: 355-372.

CHARLe, C. (1994) - La République des universitaires, 1870-1940. Seuil, Paris.

Claval, P.; A-L. SANGUIN (1995) - La géographie française à l'époque classique. L'Harmattan, Paris.

GARCÍA RAMON, M-D.; J. NOGUE I FONT; A. ALBET I MAS (1992) - La práctica de la geografía en España. Oikos-Tau, Barcelone.

GómEZ MENDOZA, J. (1997) - La formación de la Escuela Española de Geografía (1940-1952). Instituciones, revistas, congresos y programas. Eria, 42: 107-146.

HAMELIN, L-E.; C. HAMELIN (1986) - Les carrières canadiennes de Raoul Blanchard et Pierre Deffontaines. Cahiers de Géographie du Québec: 137-150.

MonBeIG, P. (1957) - "Les géographes français et l'Amérique latine”. In La géographie française au milieu du XX⿳亠丷厂 siècle, Paris: 249-256.

RoBIC, M.C. et al., (1996) - Géographes face au monde, L'Harmattan, Paris.

SERMET, J. (1957) - Les géographes français et l'Europe. In La géographie française au milieu du XX siècle, Paris: 197-210.

THERY, H.; M. DOURLENS (1991) - Pierre Monbeig. Un géographe pionnier, IHEAL, Paris.

VILA VALENTI, J. (1978) - Sur l'œuvre en Catalogne de Pierre Deffontaines (1894-1978). Revue géographique des Pyrénées et du Sud-Ouest: 527-533. 